Bundesgesundheitsbl - Gesundheitsforsch Gesundheitsschutz 2009 · 52:176-182 DOI 10.1007/s00103-009-0759-y Online publiziert: 6. Februar 2009 (c) Springer Medizin Verlag 2009

\author{
A. Ammon ${ }^{1}$ D. Faensen ${ }^{2}$ \\ ${ }^{1}$ European Centre for Disease Prevention and Control, Schweden . ${ }^{2}$ Robert-Koch- \\ Institut, Berlin, BRD
}

\title{
Surveillance von Infektionskrankheiten auf europäischer Ebene
}

Organisiert und kofinanziert wurden sie jeweils von einer Institution in einem der EU-Mitgliedsstaaten. In der Regel wurde versucht, zur jeweiligen Krankheit oder Krankheitsgruppe je einen nationalen Experten aus den Bereichen Epidemiologie und Labor aus möglichst vielen MS zusammenzubringen, um durch die Zusammenführung von Daten einen „europäischen Mehrwert" zu erzielen. Dieser kann sich zum einen in einer Standardisierung der verwendeten epidemiologischen Erhebungsmethoden oder der angewandten Labormethoden zeigen, zum anderen aber auch in der Identifikation von Länder übergreifenden Ausbrüchen liegen, die andernfalls nicht oder später entdeckt worden wären. Insbesondere durch die Standardisierung von und dem Training in Labormethoden haben diese Netzwerke auch zur Verbesserung der Surveillance in den MS beigetragen (siehe [7]).

Diese Organisationsstruktur und Art der Förderung brachten jedoch eine Reihe von Nachteilen mit sich, die mit jedem neu errichteten Netzwerk evidenter wurden:

- nicht alle DSNs deckten die gesamte $\mathrm{EU} \mathrm{ab}$,

- die geförderten DSNs bearbeiteten nur einen Teil der vom Gemeinschaftsnetz nach und nach zu erfassenden 49 übertragbaren Krankheiten und Gesundheitsrisiken,

- jedes DSN besaß eine eigene Datenbank zur Erfassung. Dies bedeutete jeweils einen hohen finanziellen und personellen Aufwand für die Entwicklung und Erhaltung der technischen Infrastruktur,

- die Surveillance-Daten eines MS mussten an viele verschiedene DSN gesendet werden, die unterschiedliche Datenaustauschformate und Variablendefinitionen vorgaben. Daraus resultierte ein erheblicher Aufwand sowohl für die Datenlieferanten in den MS als auch für die DSN,

- um EU-weite Surveillance-Daten zu erhalten, mussten verschiedene DSN konsultiert werden,

- trotz der Standardisierungsbestrebung der einzelnen DSN war eine übergreifende Vergleichbarkeit der Daten auf EU-Ebene nur sehr bedingt gegeben,

- da die Förderung nur maximal 3 Jahre betrug, musste jeweils ein Folgeantrag gestellt werden, sodass die Kontinuität nicht immer gewahrt werden konnte.

Die Grenzen dieser Surveillance, die auf einem Netzwerk der Netzwerke basiert, wurden sehr deutlich, als im Frühjahr 2003 weltweit erstmalig SARS-Erkrankungen auftraten. Das zu diesem Zeitpunkt existierende System konnte die Erwartungen an eine Koordinierung der Erfassung nicht erfüllen. Innerhalb der Jahresfrist wurde durch das Europäische Parlament und den Rat die Errichtung des Europäischen Zentrums für die Prävention und die Kontrolle von Krankheiten (European Centre for Disease Prevention 


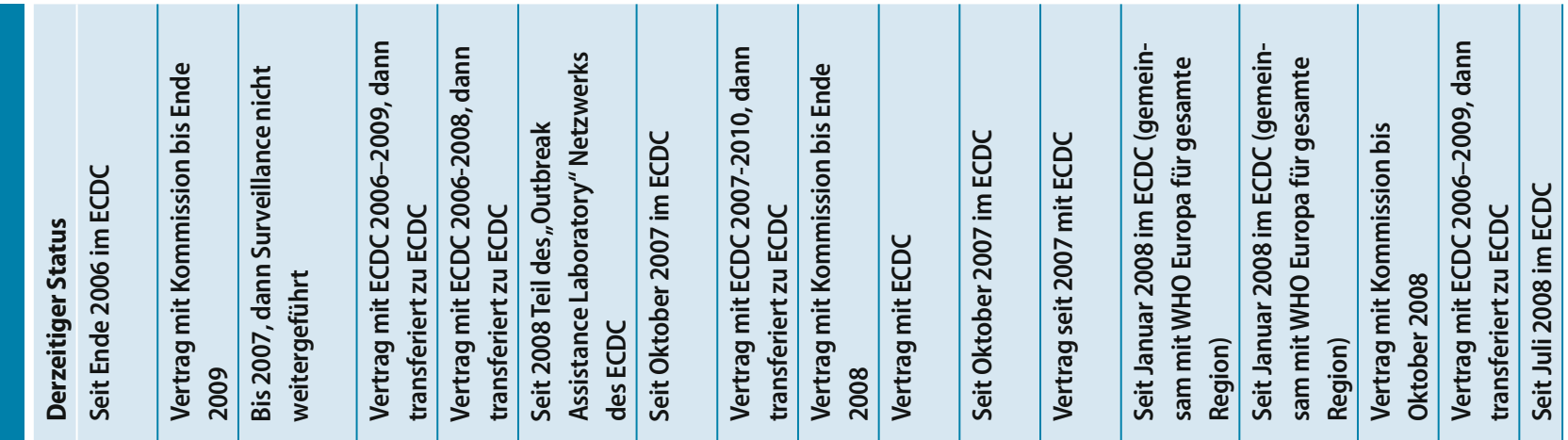

흘 
and Control, ECDC) verabschiedet [8], das am 20. Mai 2005 seine Arbeit aufnahm. Das Europäische Zentrum hat keinerlei regulative Macht, sondern soll im Wesentlichen koordinierend wirken.

Eine der Hauptfunktionen des ECDC besteht darin, die europäische Infektionsüberwachung zu standardisieren. Dabei sollen die etablierten Strukturen weiterentwickelt werden. Insbesondere sollen die Verfahrensweisen der bisher weitgehend voneinander unabhängigen Surveillance-Netzwerke für die verschiedenen Krankheiten vereinheitlicht und vom Zentrum koordiniert werden. Die im Rahmen der DSN bereits etablierten Kooperationen zwischen Laboratorien in den MS sollen weiterentwickelt und auf zusätzliche Erreger ausgedehnt werden, da das Zentrum keine eigenen Laboratorien besitzt. Ziel der Erfassung von Surveillance-Daten am ECDC ist es, diese zu analysieren, zu interpretieren und die Ergebnisse den Entscheidungsträgern in den MS, dem EU-Parlament, der Kommission und anderen Organisationen zeitnah und in geeigneter Form zur Verfügung zu stellen.

\section{Entwicklung einer EU-weiten einheitlichen Surveillance}

Das ECDC hat kürzlich die Strategie für die zukünftige Surveillance in der EU veröffentlicht [9] (web-link zum Dokument: http://ecdc.europa.eu/documents/pdf/ Surveillance_of_CD_EU.pdf). In diesem Dokument wird unterschieden zwischen der traditionellen, auf Indikatoren basierenden Surveillance (indicator-based surveillance) und der Überwachung von $\mathrm{Me}$ dien und Internet auf Ereignisse, die auf Infektionskrankheiten hindeuten (eventbased surveillance). Im Folgenden wird die Entwicklung der EU-weiten Indicatorbased-Surveillance beschrieben.

Das zukünftige EU-Surveillance-System, TESSy (The European Surveillance System) hat zum Ziel, die EU-weite Erfassung, Validierung und Verbreitung von Surveillance-Daten der EU-Mitgliedsstaaten, Islands, Liechtensteins und Norwegens zu verbessern. Das übergeordnete Bestreben ist es, die derzeitige Komplexität $\mathrm{zu}$ vereinfachen. $\mathrm{Zu}$ diesem Zweck wird die Datenerfassung standardisiert, alle Daten werden an eine Stelle geliefert und können von dieser auch wieder abgerufen werden.

Zur Nutzung und Publikation der TESSy-Daten wird mit den Mitgliedsstaaten eine Vereinbarung geschlossen werden, in der die Rechte, Pflichten und Rollen der Datenübermittler und Datennutzer festgelegt werden.

\section{Evaluation der Surveillance- Netzwerke}

Da die einzelnen Netzwerke als Projekte unabhängig voneinander beantragt wurden, haben sich zwangsläufig uneinheitliche Verfahrensweisen entwickelt (hinsichtlich der inhaltlichen Schwerpunkte, der Verfahrensweisen zur Erfassung und Auswertung der Daten sowie zur Information der Netzwerkpartner und der weiteren Fachöffentlichkeit).

Das ECDC hat daher in enger Zusammenarbeit mit Experten der MS ein Protokoll zur Evaluation und Bewertung dieser EU-Netzwerke entwickelt. Dabei wurde auf die Ergebnisse eines früheren Projekts der Kommission aufgebaut [10]. Durch diesen Prozess sollen Aktivitäten der Netzwerke identifiziert werden, die die zukünftige Surveillance auf EU-Ebene unterstützen. Die Evaluation erstreckt sich auf den Nutzen des Netzwerks, die Qualität der technischen Ausführung und die Erfüllung der im Vertrag mit der Kommission genannten Zielstellungen. Neben der Auswertung einer umfangreichen Dokumentation über das jeweilige Netzwerk werden den Evaluations-Teams die Ergebnisse standardisierter Nutzerbefragungen (das sind die epidemiologischen und mikrobiologischen Netzwerkpartner und die nationalen Surveillance-Koordinatoren) zur Verfügung gestellt. Ein Besuch beim koordinierenden Institut mit ergänzenden Interviews der Mitarbeiter der Koordinationsstelle rundet die Informationserfassung für die Evaluation ab. Im Bericht nimmt das Evaluationsteam Stellung zu den Stärken und Schwächen des Netzwerks und beschreibt, welche Komponenten gut etabliert bzw. noch entwicklungsbedürftig sind. Mit einem speziell erstellten Bewertungsinstrument beurteilen die Evaluationsteams abschließend, inwieweit die Netzwerkaktivitäten mit der zukünf- tigen EU-Surveillance kompatibel sind. $\mathrm{Zu}$ diesem Zweck wird auf die generellen Kriterien für Ziele einer international ausgerichteten Surveillance aus der Arbeit von Ruutu et al. Bezug genommen [11]. In der Beurteilung geben die Teams ihre Meinung darüber ab, welche Netzwerkaktivitäten weitergeführt, modifiziert und beendet werden sollten, ob das ECDC die Koordination übernehmen oder ob die Aktivitäten nach außen vergeben werden sollten. Die Instrumente für die Evaluation und Bewertung werden nach Abschluss aller Netzwerk-Evaluationen veröffentlicht (voraussichtlich 2. Quartal 2009).

Da sich der Prozess der Evaluation aller Surveillance-Netzwerke über mehr als 3 Jahre erstreckt (bedingt durch die unterschiedlichen Auslaufzeitpunkte der Verträge), wurde ein Lenkungsausschuss gegründet, der den Prozess begleitet. Seine Mitglieder setzen sich aus Vertretern der Kommission, dem Europäischen Parlament, der WHO, den Centers for Disease Control and Prevention (CDC) und dem Verwaltungsrat des ECDC zusammen.

Die Entscheidung des ECDC über die Fortführung der Netzwerke basiert auf mehreren Komponenten: Priorität der jeweils bearbeiteten Erkrankung(en), Ergebnis der Evaluation, ausreichende Personalkapazität im ECDC vorhanden, Kosteneffektivität. Für den Transfer der Koordinationstätigkeit an das ECDC wird mit den Netzwerk-Koordinatoren ein Transferplan ausgearbeitet, der alle Elemente des jeweiligen Netzwerks berücksichtigt. Er umfasst u. a. den Transfer der Datenbanken und Webseiten-Inhalte, einen orientierenden Besuch von ECDCMitarbeitern bei der Koordinationsstelle, um die täglichen Arbeitsabläufe kennenzulernen, und die Vorbereitung von Ausschreibungen, z. B. für Labortätigkeiten. Die Netzwerk-Mitglieder in den MS werden über die Veränderungen informiert und insbesondere in die Diskussion um eine Weiterentwicklung der bisherigen Surveillance mit einbezogen. Die Daten der Netzwerke werden in die neue Datenstruktur des TESSy überführt und in die Datenbank importiert. Die Nutzer werden spezifisch geschult. 
TESSy

Im Jahr 2006 wurde mit der Entwicklung von TESSy begonnen. Sein Kernstück ist ein System, das den Nutzern eine einheitliche Schnittstelle zur Übermittlung und Nutzung europaweiter Surveillance-Daten bietet.

Im Februar 2007 wurde vom ECDC ein Treffen der potenziellen TESSy-Nutzer organisiert. Eingeladen waren je ein Vertreter der Epidemiologie und des Datenmanagements aus allen EU-/EEA-Mitgliedsstaaten sowie aus den DSN. Ziele dieses Treffens waren die Identifikation von Anforderungen an TESSy sowie die Entwicklung von Vereinbarungen zur Datennutzung. Seine Ergebnisse sind in [12] dokumentiert. Sie wurden weitestgehend bei der Entwicklung des TESSy und dem Wechsel der DSNs an das ECDC berücksichtigt.

Innerhalb von TESSy wird für alle Krankheiten unter EU-Surveillance ein limitierter Satz von Standardvariablen erfasst. Für Krankheiten mit hoher Priorität werden zusätzliche Informationen (hinsichtlich der Labormerkmale, Informationen über Reisen in der Inkubationszeit, Verhaltensparameter etc.) erhoben.

TESSy ist so konzipiert, dass jederzeit durch Änderungen von Metadaten (also von Daten, die die Datenstruktur beschreiben) neue Variablen zu bestehenden Variablensätzen hinzugefügt oder auch ganz neue Variablensätze definiert werden können, etwa wenn zusätzliche Information bzw. neue Erreger oder Krankheiten in die Surveillance aufgenommen werden sollen. Die Datenaustauschformate sind in TESSy vereinheitlicht. Es werden 2 Formate unterstützt: ein vergleichsweise einfaches Format mit kommaseparierten Werten (CSV-Format), das aus den meisten Datenbanken oder Kalkulationsprogrammen exportiert werden kann, sowie ein XML-Format ${ }^{1}$. Letzteres erfordert zwar eine gewisse Expertise bei der Erstellung, es ist jedoch flexibler und unterstützt Strukturen, die in einem CSV-Format nicht möglich sind, wie zum Beispiel wiederholbare Datenfelder. Die neuen For-

${ }^{1} \mathrm{XML}=$ eXtensible Markup Language. World Wide Web Consortium: Extensible Markup Language (XML). http://www.w3.org/XML/

Bundesgesundheitsbl - Gesundheitsforsch - Gesundheitsschutz 2009 - 52:176-182 DOI 10.1007/s00103-009-0759-y

(c) Springer Medizin Verlag 2009

\section{A. Ammon · D. Faensen}

\section{Surveillance von Infektionskrankheiten auf europäischer Ebene}

\section{Zusammenfassung}

Mit der im Jahr 1998 von EU-Parlament und Ministerrat getroffenen Entscheidung

zur Etablierung eines EU-weiten Netzwerks zur Überwachung von Infektionskrankheiten wurde die Grundlage für die EUweite Surveillance geschaffen. Seit Mai 2005 hat das Europäische Zentrum für die Prävention und die Kontrolle von Krankheiten (ECDC) die Aufgabe, dieses Netzwerk zu koordinieren und weiterzuentwickeln. Eine der Hauptfunktionen des ECDC besteht darin, die europäische Infektionsüberwachung zu standardisieren und insbesondere die Verfahrensweisen der weitgehend voneinander unabhängigen Surveillance-Netzwerke zu vereinheitlichen. Als einer der ersten Schritte wurden in Zusammenarbeit mit den Mitgliedsstaaten die EU-Falldefinitionen für die Surveillance revidiert. Die SurveillanceNetzwerke werden derzeit nach einem standardisierten Protokoll evaluiert, bevor im ECDC eine Entscheidung über die Zukunft der einzelnen Netzwerkaktivitäten getroffen wird. Parallel wurde mit der Entwicklung eines einheitlichen Datenerfassungssystems (The European Surveillance System, TESSy) begonnen. Seit Beginn 2008 werden die Nutzer spezifisch geschult. TESSy ist seit April 2008 funktionsfähig. Zukünftig muss das Hauptaugenmerk auf die Qualität und Vergleichbarkeit der Daten gerichtet sein, da nur valide und vergleichbare Daten eine gute Grundlage für Entscheidungen im Gesundheitsbereich sind.

\section{Schlüsselwörter}

Surveillance - ECDC · Datenqualität . elektronische Datenerfassung .

Surveillance-Netzwerke

\section{Surveillance of infectious diseases at the EU level}

\section{Abstract}

The basis for EU wide surveillance was Decision 2119/98/EC of the European Parliament and the Council in 1998. Since May 2005 it is the task of the European Centre for Disease Prevention and Control to coordinate and further develop this network. One key function of the ECDC is to standardise European surveillance and especially to harmonise the procedures of the surveillance networks that developed independently of each other. As a first step, the EU case definitions have been revised jointly with the Member States and the Commission. All surveillance networks are evaluated with a standard protocol before a decision is made at the ECDC on the continuation of the individual network activities. Simultaneously, the development of The European Surveillance System (TESSy) progressed. Since the beginning of 2008 data users have been trained and TESSy has been in use since April 2008. In the future the main focus must be the improvement of the quality and comparability of the data as such data are the essential prerequisite for decision making in public health.

\section{Keywords}

surveillance $\cdot E C D C$ - data quality - electronic data collection · surveillance networks 
mate lösen alle in den DSN verwendeten Formate ab. Um den Datenlieferanten den Übergang zu erleichtern, bietet TESSy einen Konvertierungsdienst für die im Basic Surveillance Network, im EuroTB und im EuroHIV verwendeten Formate auf der TESSy-Homepage an.

Je nach Verfügbarkeit von SurveillanceDaten auf nationaler Ebene können sowohl einzelfallbasierte als auch aggregierte Daten an TESSy übermittelt werden. Beide Typen werden in einem gemeinsamen Data Warehouse zusammengefasst. Dabei werden die Surveillance-Daten mit einer Beschreibung der Datenquelle (also des Surveillance-Systems, mit dem die Daten erfasst wurden) verknüpft [13]. Damit wird das Problem der Vergleichbarkeit der Daten zwar nicht gelöst, aber zumindest entschärft. Außerdem kann die Herkunft der Daten in den Analysen angegeben werden. Diese Beschreibung umfasst zwar nur einige Schlüsseleigenschaften des Surveillance-Systems, kann aber beim Vergleich zumindest einen Hinweis darüber liefern, ob Unterschiede in den Fallzahlen oder Inzidenzen verschiedener Länder möglicherweise auf Unterschiede in den Surveillance-Systemen beruhen. In $\bullet$ Übersicht 1 sind die erfassten Eigenschaften zur Beschreibung der Datenquellen gelistet.

Zugang zu TESSy haben derzeit ausschließlich Personen, die von den zuständigen Stellen in den Mitgliedstaaten als solche nominiert wurden. Die zuständigen Stellen können das Recht zur Nominierung von Nutzern an eine Person delegieren.

Für die nominierten Nutzer organisiert das ECDC Schulungen. Die anfallenden Kosten werden vom ECDC getragen. Einige Schulungen finden am ECDC statt: Zur Auffrischung des Erlernten oder zur Vermittlung der erforderlichen Kenntnisse zur Datenaufbereitung für spezielle Krankheitsgruppen wurden und werden Online-Schulungsmaterialien vorbereitet. Außerdem werden die Nutzer zur Teilnahme an Telefonkonferenzen eingeladen. Bei Fragen und Problemen inhaltlicher und technischer Art steht ein Helpdesk zur Verfügung.

Die Datenlieferung durch die Mitgliedsstaaten soll zunächst in dem Rhythmus erfolgen, der in den jeweiligen DSN

Übersicht 1

Erfasste Eigenschaften zur Beschreibung der Surveillance-Systeme in TESSy

Gesetzliche Meldepflicht oder Sentinel

- Verwendete Falldefinition

- Geografische Abdeckung (ganzes Land oder nur Teile)

- Verfügbarkeit von Einzelfalldaten oder aggregierten Daten

- Verfügbare Informationen auf nationaler Ebene (klinische Daten, Laborbestätigung, epidemiologischer Zusammenhang)

- Wer meldet die Daten (Labor, Ärzte, Krankenhäuser ...)

- Bezug der Surveillance-Daten zu den Labordaten der nationalen Referenzzentren

- Zeitpunkt, ab wann vergleichbare Daten verfügbar sind

eingeführt war. Eine Entscheidung über die zukünftige Meldefrequenz wird im Zusammenhang mit den EU-Surveillance-Zielen für die jeweilige Erkrankung im Konsens mit den MS getroffen werden. Erste Probeläufe haben mit der Lieferung eines Basisvariablensatzes zu allen Krankheiten (in Entscheidung 2119/98) für den Jahresbericht 2006 des ECDC sowie mit der Lieferung der erweiterten Daten zu Zoonosen für den von der Europäische Behörde für Lebensmittelsicherheit (EFSA) herausgegebenen Zoonosenbericht 2007 stattgefunden.

Für die Datenlieferung wird eine XMLoder CSV-Datei (s. o.) erstellt, die die Surveillance-Daten enthält. Ein autorisierter Nutzer kann diese Datei über die TESSy-Homepage an die TESSy-Datenbank übertragen. In TESSy ist eine Reihe von Validierungsregeln hinterlegt, die nach der Datenübertragung auf die Daten angewandt werden. Der Nutzer kann das Validierungsergebnis prüfen und die übermittelten Daten anschließend freigeben. Erst dann werden sie in die EU-Datenbank aufgenommen und für andere Nutzer sichtbar.

Mittelfristig wird zusätzlich zur Datenübertragung über die TESSy-Homepage eine Maschine-zu-Maschine-Schnittstelle (Web Service) zur Verfügung stehen. Diese wird eine vollautomatische Datenlieferung ermöglichen, bei der nur im Falle eines Fehlers eine menschliche Intervention nötig ist. Datenlieferungen in kürzeren Intervallen, wie sie etwa bei Großveranstaltungen oder im Falle einer Pandemie erforderlich werden könnten, sind dann ohne nennenswerten Mehraufwand möglich.
„Daten für Taten" - Veröffentlichung und Nutzung der Daten

Die Erfassung von Surveillance-Daten auf EU-Ebene ist kein Selbstzweck. Neben der regelmäßigen Berichterstattung soll sie vor allem dazu dienen, frühzeitig Trends bei Krankheiten oder Risikofaktoren zu erkennen, früh vor Bedrohungen der Gesundheit der EU-Bürger zu warnen und insbesondere Ereignisse zu erkennen, die mehr als einen MS betreffen. Zur Nutzung der Daten steht auf der TESSy-Homepage eine Reihe von Standardberichten bereit. Regelmäßig genutzt werden die Daten für den epidemiologischen Jahresbericht des ECDC [14], dessen erste Ausgabe 2007 zum ersten Mal eine Übersicht über Trends bei allen unter EU-Surveillance stehenden Krankheiten präsentiert. Auch für die Humandaten im Europäischen Zoonosenbericht, den die EFSA jährlich veröffentlicht, werden die Daten aus TESSy durch das ECDC zur Verfügung gestellt [15]. WHO Europa und das ECDC führen die gemeinsame Surveillance von HIV/AIDS nach dem Vertragsende von EuroHIV gemeinsam weiter. Dabei dient TESSy als gemeinsame Datenbank.

\section{Rolle Deutschlands}

Deutschland ist beziehungsweise war in den meisten DSN als Mitglied vertreten. Von Deutschland aus wurde aber nur ein Netzwerk koordiniert, das European Network for Diagnostics of „imported“ Viral Diseases (ENIVD, siehe dazu auch $\bullet$ Tabelle 1).

Die Surveillance-Daten, die aus Deutschland an die DSN und nachfolgend 
an TESSy übermittelt werden, entstammen unterschiedlichen Datenquellen. In den meisten Fällen werden jedoch Daten der nach dem Infektionsschutzgesetz (IfSG) erhobenen Fälle meldepflichtiger Infektionserreger gesendet. An einige Netzwerke wurden alternativ oder zusätzlich Labordaten aus den Nationalen Referenzzentren und Konsiliarlaboratorien geliefert. Wie eingangs erwähnt, mussten zu diesem Zweck mehrere unterschiedliche Datenschnittstellen bedient werden. Die Entwicklungen, die sich durch die Implementierung von TESSy als zentraler Datenquelle für europäische SurveillanceDaten abzeichnen, werden daher zu einer erheblichen Entlastung aufseiten der Datenlieferanten führen. Am Robert KochInstitut (RKI) wurden Routinen entwickelt, um die Daten im einheitlichen XML-Format aufzubereiten. Die direkte Datenlieferung an TESSy wird nach und nach die Lieferung an die DSN in unterschiedlichen Formaten ablösen. Langfristig soll auch in Deutschland die Lieferung vollautomatisch über den TESSy-WebService erfolgen.

Bei der durch das ECDC koordinierten Revision der EU-Falldefinitionen wurde auf die Erfahrungen in Deutschland zurückgegriffen. Hier wurden die 2001 veröffentlichten Falldefinitionen in einem Ringversuch auf ihre Eindeutigkeit und Anwendbarkeit getestet [16]. Basierend auf den Ergebnissen dieser Studie wurde für die am 1.1.2004 in Kraft getretenen Falldefinitionen eine neue, für alle Übermittlungskategorien einheitliche Struktur festgelegt $[17,18]$. Außerdem wurde auf eindeutige Formulierungen geachtet.

Für die EU-Falldefinitionen wurde eine an das deutsche Muster angelehnte Struktur verwendet. Der erste Entwurf der neuen EU-Falldefinitionen wurde vom ECDC den zuständigen Stellen in den MS mit der Bitte um Kommentierung zur Verfügung gestellt. In Deutschland wurden die Entwürfe am RKI begutachtet und zum Teil ausführlich kommentiert. Die Kommentare der MS wurden vom ECDC zusammengetragen und nach Möglichkeit eingearbeitet. Widersprüche in den Kommentaren wurden - wenn möglich - aufgelöst. Die Revision wurde im zweiten Anlauf durch das NetzwerkKomitee akzeptiert und am 28.4.2008 ver- öffentlicht [4]. Es überrascht nicht, dass hierbei einige in Deutschland geltende Kriterien nicht in die EU-Falldefinitionen übernommen werden konnten. So verlangt beispielsweise die aktuelle EU-Falldefinition für Syphilis einen Nachweis von Tp-IgM-Antikörpern zur Erfüllung der Laborkriterien, wenn kein direkter Erregernachweis vorliegt. Ein solcher ist für die Syphilisfälle in Deutschland aber nicht regelmäßig vorhanden und ist auch nicht Bestandteil der deutschen Falldefinition. Es ist angestrebt, solche Inkompatibilitäten zu beseitigen.

\section{Diskussion und Ausblick}

Mit der Entwicklung des europäischen Surveillance-Systems TESSy wurde eine einheitliche Schnittstelle für die Lieferung von Surveillance-Daten geschaffen, die den EU-Mitgliedsstaaten und weiteren Ländern in Zukunft die Arbeit erheblich erleichtern wird. Mit dem Übergang der DSN an das ECDC wird TESSy die zentrale Quelle für Surveillance-Daten auf EUEbene sein. Derzeit können die Anwender nur auf vordefinierte Tabellen und Berichte zugreifen, die zwar täglich aktualisiert werden, jedoch keine Flexibilität bei der Auswahl von Variablen, Gruppierungen usw. bieten. Es ist geplant, eine Abfrageschnittstelle zu entwickeln, bei der abhängig von den Berechtigungen des Anwenders beliebige Variablen kombiniert werden können - ähnlich wie dies für die Meldedaten nach IfSG in Deutschland durch SurvStat@RKI (http://www3. rki.de/SurvStat) möglich ist.

Der Zugang zu TESSy ist derzeit nur autorisierten Nutzern vorbehalten. Die Allgemeinheit und Fachöffentlichkeit erhalten gegenwärtig nur aufbereitete und kommentierte Daten in Form von Berichten und anderen Publikationen des ECDC und dessen Partnerorganisationen. Es wäre zu begrüßen, wenn ein Teildatensatz frei zugänglich gemacht werden könnte. Dies könnte unter anderem die wissenschaftliche Nutzung der Daten stimulieren. Außerdem könnte die Fachöffentlichkeit viele Fragen eigenständig beantworten, was zur Entlastung der Experten in den nationalen und internationalen Surveillance-Instituten führen würde - eine Erfahrung, die am RKI bereits mit der
Einführung von SurvStat@RKI gemacht wurde. Planungen in dieser Richtung bestehen, jedoch muss hier ein Konsens zwischen allen Dateneigentümern gefunden werden.

Zur Detektion von Ausbrüchen sollen unter anderem Algorithmen eingesetzt werden, die insbesondere solche Ereignisse anzeigen sollen, die auf nationaler Ebene nicht erkennbar sind, z. B. aufgrund mangelnder Ressourcen oder weil in einzelnen Ländern nur Einzelfälle auftreten. Dies spielt insbesondere bei lebensmittelbedingten Infektionen, aber z. B. auch bei reiseassoziierten Legionellosen eine Rolle.

Gemäß Zoonosenrichtlinie [19] sind die EU-Mitgliedsstaaten verpflichtet, Daten zu Ausbrüchen an die EFSA zu übermitteln (siehe hierzu auch den Beitrag von H. Wichmann-Schauer et al. im vorliegenden Heft). In Deutschland sind die Meldungen zu Ausbrüchen und Fällen von Infektionskrankheiten nach IfSG im Meldesystem eng miteinander verknüpft [20]. Es wäre daher zu begrüßen, wenn in Zukunft Ausbrüche und Einzelfälle an das TESSy gemeldet würden, sodass das ECDC diese dann im Auftrag der Mitgliedstaaten an die EFSA weiterleiten könnte. Die integrierte Meldung von Ausbrüchen bietet auch einen Mehrwert für die automatisierte Ausbruchserkennung, da hier bereits erkannte und bearbeitete Ausbrüche aus den generierten Signalen ausgefiltert werden könnten.

Ein Problem der internationalen Surveillance ist die Vergleichbarkeit der Daten. Verschiedene Länder haben sehr unterschiedliche Surveillance-Systeme. Sie unterscheiden sich in Bezug auf die Meldepflichten, es sind zum Teil Systeme eingerichtet, bei denen ausgewählte Einrichtungen der Gesundheitsvorsorge oder -versorgung Surveillance-Daten erheben (Sentinel-Systeme). Weitere Einschränkungen bei der Datenvergleichbarkeit resultieren aus der unterschiedlichen Bereitschaft der Ärzte zur Durchführung einer Labordiagnostik und der Meldepflichtigen zur Einhaltung der Meldepflicht. Gründe sind auch Unterschiede in den Systemen der Krankenversicherungen und im Verhalten der Patienten (Health Care Seeking Behaviour). Langfristig soll die Vergleichbarkeit verbessert werden. 
Erste diesbezügliche Schritte sind die Verknüpfung der Daten mit den Beschreibungen der Systeme zur Datenerfassung sowie die Neufassung der Falldefinitionen, die von den Mitgliedsstaaten umgesetzt werden sollen. Weiterhin wird versucht, die Untererfassung in den verschiedenen Ländern zu quantifizieren und die Dunkelziffer bei den Meldungen zu erhellen. Auch wenn dies noch ein hartes Stück Arbeit sowohl für das ECDC als auch für die Mitgliedsstaaten bedeutet, sind sich alle einig, dass nur valide und vergleichbare Daten eine gute Grundlage für Entscheidungen im Gesundheitsbereich sowohl auf nationaler als auch auf EU-Ebene sind.

\section{Danksagung}

Unser besonderer Dank gilt Frau Dr. Poggensee für die Informationen zur Rolle Deutschlands bei der Revision der EUFalldefinition, Herrn Dr. Claus für die Durchsicht des Manuskripts sowie dem TESSy-Team für seinen Einsatz bei der Entwicklung des europäischen Surveillance-Systems.

\section{Korrespondierende Autorin}

\section{Dr. Andrea Ammon, MPH}

European Centre for Disease Prevention and Control (ECDC)

17183 Stockholm, Schweden

E-Mail: Andrea.Ammon@ecdc.europa.eu

\section{Literatur}

1. Entscheidung 2119/98/EC des Europäischen Parlaments und des Rates vom 24. September 1998 über die Schaffung eines Netzes für die epidemiologische Überwachung und die Kontrolle übertragbarer Krankheiten in der Gemeinschaft. OJ L268, 3.10.1998, S 1-6

2. Entscheidung $2000 / 96 / E G$ der Kommission vom 22. Dezember 1999 betreffend die von dem Gemeinschaftsnetz nach und nach zu erfassenden übertragbaren Krankheiten. OJ L28, 3.2.2000, S 50-53

3. Entscheidung 2003/534/EG der Kommission vom 17. Juli 2003 zur Änderung der Entscheidungen 2119/98/EG, 2000/96/EG betreffend der aufgelisteten Infektionskrankheiten und 2002/253/EG betreffend der Falldefinitionen. OJ L184, 23.7.2003, S 35-39

4. Entscheidung 2008/426/EC der Kommission vom 28. April 2008 zur Änderung der Entscheidung 2002/253/EG der Kommission zur Festlegung von Falldefinitionen für die Meldung übertragbarer Krankheiten an das Gemeinschaftsnetz gemäß der Entscheidung 2119/98/EG des Europäischen Parlaments und des Rates. OJ L159, 18.6.2008, S 46-90
5. Entscheidung 2002/253/EC der Kommission vom 19. März 2002 zur Festlegung von Falldefinitionen für die Meldung von Infektionskrankheiten an das Gemeinschaftsnetz. OJ L86, 3.4.2002, S 44-62

6. Entscheidung 2003/542/EG der Kommission vom 17. Juli 2003 zur Änderung der Entscheidung 2000/96/EG betreffend die Betreibung spezieller Überwachungsnetze. OJ L185, 24.7.2003, S 55-58

7. Fisher IST on behalf of the Enter-net participants (1999) The Enter-net international surveillance network - how it works. Euro Surveill 4:52-55

8. Verordnung (EG) Nr. 851/2004 des Europäischen Parlaments und des Rates vom 21. April 2004 zur Errichtung eines Europäischen Zentrums für die Prävention und die Kontrolle von Krankheiten. OJ L142, 30.4.2004, S 1-11

9. Amato-Gauci A, Ammon A (2008) The surveillance of communicable diseases in the European Union - a long-term strategy (2008-2013). Euro Surveill 13 (26):pii=18912. Available online: http://www. eurosurveillance.org/ViewArticle.aspx?Articleld= 18912

10. Protocol for the evaluation of EU-wide surveillance networks on communicable diseases, Kansanterveyslaitos Folkhälsoinstitutet Finland, 2004 http:// ecdc.europa.eu/documents/surveillance/Surveval \%202004.pdf

11. Ruutu P, etal. (2001) A framework and recommendations for evaluating surveillance systems within the community network for communicable diseases. European Commission: Directorate General Health \& Consumer protection. Agreement No. S12.300119 (2000CVG4-030), 2001, http://ecdc. europa.eu/documents/surveillance/Conceptual_ Framework\%20Ruutu\%20Feb\%202001\%20(3).pdf

12. ECDC: First Meeting of the TESSy Working Group. Stockholm, 14-15 February 2007 http://ecdc. europa.eu/pdf/TESSy_workgroup.pdf

13. Rolfhamre $P$, Faensen $D$, van Straten $E, A m m o n ~ A$ (2007) The European Surveillance System (TESSy) - strengthening the infectious disease surveillance in Europe. European Scientific Conference on Applied Infectious Disease Epidemiology ESCAIDE Stockholm, 1-20 October 2007, Poster 14.23

14. European Communicable Disease Epidemiological Report. http://www.ecdc.europa.eu/pdf/ECDC epi_report_2007.pdf

15. The Community Summary Report on Trends and Sources of Zoonoses, Zoonotic Agents, Antimicrobial resistance and Foodborne outbreaks in the European Union in 2006. http://www.efsa.europa. eu/EFSA/efsa_locale-1178620753812 1178671312912.htm

16. Krause G, Brodhun B, Altmann D, etal. (2006) Reliability of case definitions for public health surveillance assessed by Round-Robin test methodology. BMC Public Health 6:129

17. Benzler J, etal. (2004) Zur Ausgabe 2004 der Falldefinitionen für die Surveillance meldepflichtiger Infektionskrankheiten in Deutschland. Bundesgesundheitsbl Gesundheitsforsch Gesundheitsschutz 47:141-146

18. Poggensee G, etal. (2006) Falldefinitionen für die Surveillance meldepflichtiger Infektionskrankheiten in Deutschland, Ausgabe 2007. Bundesgesundheitsbl Gesundheitsforsch Gesundheitsschutz 49:1189-1194

19. Richtlinie 2003/99/EG des Europäischen Parlaments und des Rates vom 17. November 2003 zur Überwachung von Zoonosen und Zoonoseerregern und zur Änderung der Entscheidung 90/424/ EWG des Rates sowie zur Aufhebung der Richtlinie 92/117/EWG des Rates: OJ L 325, 12.12.2003, S $31-40$
20. Krause G, Altmann D, Faensen D, et al. (2007) SurvNet electronic surveillance system for infectious disease outbreaks, Germany. Emerg Infect Dis 13:1548-1555 